\title{
60 Jahre Thomas-Mann-Archiv der ETH Zürich: ein Bericht über die Jubiläumstagung vom 1. September 2016
}

\author{
Journal Article \\ Author(s): \\ Wiederkehr, Stefan (D) \\ Publication date: \\ 2017-02
}

Permanent link:

https://doi.org/10.3929/ethz-b-000249401

Rights / license:

Creative Commons Attribution-NonCommercial-ShareAlike 4.0 International

Originally published in:

Zeitschrift für Bibliothekswesen und Bibliographie 64(1), https://doi.org/10.3196/186429501664160 


\section{Berichte}

\section{Jahre Thomas-Mann-Archiv der ETH Zürich}

Ein Bericht über die Jubiläumstagung vom 1. September 2016

D ie ETH Zürich feierte das 60-jährige Bestehen des Thomas-Mann-Archivs mit einer internationalen Tagung, die am 1. September 2016 in der voll besetzten historischen Semper Aula stattfand. Gegenstand der Tagung waren die Beziehungen Thomas Manns zu prominenten jüdischen Kollegen, namentlich Max Brod, Franz Kafka, Jakob Wassermann, Samuel Fischer und Stefan Zweig.

\section{Ein literarischer Nachlass an der ETH Zürich}

Der stellvertretende Direktor der ETH-Bibliothek, Andreas Kirstein, rief in seiner Begrüßung in Erinnerung, wie der Literaturnobelpreisträger Thomas Mann (18751955) und die ETH Zürich vor 60 Jahren zueinander gefunden hatten: Nach der Machtergreifung der Nationalsozialisten unternahm Thomas Mann 1933 eine Vortragsreise ins Ausland und entschloss sich in der Schweiz, nicht mehr nach Deutschland zurückzukehren. Die positiven Erinnerungen an die Jahre des Exils in Zürich (1933-1938) bewogen ihn, erneut dort Wohnsitz zu nehmen, als er 1952 aus den USA nach Europa zurückkehrte.

Das Thomas-Mann-Archiv der ETH Zürich wurde 1956 auf Initiative des damaligen Rektors, Karl Schmid, eingerichtet. Der Germanist Schmid war bereits die treibende Kraft hinter der Verleihung eines Ehrendoktors der Naturwissenschaften zu Thomas Manns 80. Geburtstag 1955 gewesen. Der Jubilar hatte hocherfreut auf diese Ehrung reagiert, so dass die Einrichtung eines Archivs an der ETH Zürich nahe lag, als er nur zwei Monate später starb und die Erben eine Schweizer Institution von nationaler Bedeutung suchten, die die langfristige Sicherung des Nachlasses garantieren konnte.

Die Annahme der Schenkung des literarischen Nachlasses von Thomas Mann fügte sich ein in das Bestreben der ETH Zürich, nicht nur Fachspezialisten zu schulen, sondern verantwortungsvoll handelnde Persönlichkeiten auszubilden, die gesellschaftliche und ethische Aspekte ihrer Tätigkeit als Ingenieur oder Naturwissenschaftler reflektieren. Diese Zielsetzung begleitete die ETH Zürich seit ihrer Gründung. Der Nachlass Thomas Manns besteht aus Werkmanuskripten, Tagebüchern, Korrespon- denz, Fotografien, der privaten Bibliothek, der Einrichtung seines letzten Arbeitszimmers sowie einer umfangreichen Pressedokumentation über ihn. Seit 1956 wurden diese unikalen Bestände durch weitere Schenkungen und Ankäufe ergänzt sowie eine Spezialbibliothek mit einschlägiger Forschungsliteratur aufgebaut. Das ThomasMann-Archiv kann daher für sich in Anspruch nehmen, die wichtigste Einrichtung zur Erforschung von Leben und Werk Thomas Manns weltweit zu sein. ${ }^{1}$

Im Zuge einer Reorganisation wurde das ThomasMann-Archiv 2012 innerhalb der ETH Zürich der ETH-Bibliothek zugeordnet. Dies bot unter anderem die Chance, informationswissenschaftlich neue Wege zu beschreiten. Nach einem mit großem Aufwand und Engagement vorangetriebenen Erschließungs- und Digitalisierungsprojekt gehört das Thomas-Mann-Archiv seit $2015 \mathrm{zu}$ den ganz wenigen Archiven weltweit, deren Gesamtbestand auf Ebene der Einzeldokumente in einem modernen Archivinformationssystem erschlossen und vollständig digitalisiert ist. Die Erschließungsdaten des Thomas-Mann-Archivs sind heute über das Web frei verfügbar. ${ }^{2}$ Einer Freischaltung der Digitalisate stehen hingegen noch rechtliche Hindernisse entgegen. ${ }^{3}$

Auch der Präsident des Kuratoriums, Andreas Kilcher, hob hervor, wie sehr das Thomas-Mann-Archiv vom Wechsel zur ETH-Bibliothek und von der Neubesetzung der Archivleitung 2012 profitiert hat. Dies betrifft nicht nur informationswissenschaftliche Innovation, sondern auch die literaturwissenschaftliche Forschung. Das zeigen die bereits abgeschlossenen Projekte ebenso wie das aktuell laufende Projekt zu Thomas Manns Nachlassbibliothek. Wie sein Vorredner Kirstein und später die Leiterin des Thomas-Mann-Archivs, Katrin Bedenig, unterstrich Kilcher die Weitsicht der Familie Mann. Im Vergleich etwa mit dem Nachlass Max Brods, der in privater Hand der Forschung entzogen blieb, mit wirtschaftlichen Interessen in kleinen Portionen teilveräußert wurde und als Gegenstand von Eigentumsstreitigkeiten noch nach Jahrzehnten das Oberste Gericht Israels beschäftigte, nimmt sich die Regelung zwischen Erben und Institution im Falle des Thomas-Mann-Nachlasses geradezu mustergültig aus. 
Die Leiterin des Thomas-Mann-Archivs, Katrin Bedenig, wies in ihren Dankesworten auf den langjährigen fruchtbaren Austausch des Thomas-Mann-Archivs mit zahlreichen Literaturarchiven sowie weiteren Institutionen im In- und Ausland hin. Dazu gehören auch die partnerschaftliche Zusammenarbeit mit Verlagen und natürlich die Kooperation innerhalb der ETH Zürich. So ist das größte aktuelle Forschungsvorhaben am ThomasMann-Archiv ein gemeinsam vor der Professur Kilcher und der ETH-Bibliothek durchgeführtes, vom Schweizerischen Nationalfonds gefördertes Projekt zur Erschließung, digitalen Präsentation und Erforschung der Marginalien und Lesespuren in Thomas Manns Nachlassbibliothek. ${ }^{4}$ In ihrer Einführung zum Tagungsgegenstand charakterisierte Bedenig Thomas Manns Verhältnis zur jüdischen Thematik als vielfältig und höchst ambivalent. Der spätere Nobelpreisträger scheint die Gefahr antisemitischer Vorurteile lange unterschätzt zu haben. Er trug in den Jahren 1895 und 1896 zur deutlich antisemitischen Zeitschrift Das Zwanzigste Jabrbundert bei, ließ in seinen Erzählungen und Romanen stereotyp gezeichnete jüdische Figuren auftreten und veröffentlichte eine Reihe essayistischer Beiträge zur jüdischen Thematik, die nicht frei von Widersprüchen und zeitgenössischen Stereotypen sind. Dem stehen später aber umso deutlichere öffentliche Stellungnahmen gegen den nationalsozialistischen Antisemitismus gegenüber. Auch die Arbeit am Romanwerk Joseph und seine Brüder, worin er den alten Hebräern ein literarisches Denkmal setzte, führte Thomas Mann nach der nationalsozialistischen Machtergreifung fort und veröffentlichte während des Exils bis 1943 sämtliche Bände der Tetralogie. Der intellektuelle und persönliche Austausch mit jüdischen Kollegen gehörte zu den prägenden Einflüssen auf Thomas Mann, was dieser 1921 mit scherzhaftem Unterton so kommentierte: »Juden haben mich sentdeckt<, Juden mich verlegt und propagiert [...]. Und wenn ich in die Welt gehe, Städte bereise, so sind es, nicht nur in Wien und Berlin, fast ohne Ausnahme Juden, die mich empfangen, beherbergen, speisen und hätscheln«.

\section{Mann, Kafka und Brod}

Die beiden Vorträge von Andreas Kilcher (Zürich) über Max Brod (1884-1968) und Thomas Mann sowie von Jutta Linder (Messina) über Thomas Mann als Kafka-Leser ergänzten sich gegenseitig, waren doch KafkaLektüre und -Edition ein wesentlicher Gegenstand des Austauschs zwischen Max Brod und Thomas Mann. In Abgrenzung von den älteren Lesarten Hans Wyslings und Mark Gelbers, die beide - wenn auch mit konträrem Ergebnis - das Verhältnis von Thomas Mann und Brod strukturell aus dem deutsch-jüdischen Zusammenhang gedeutet hatten, fragte Kilcher nach dem Individuellen, also nach der persönlichen Beziehung von Thomas Mann und Max Brod. Aus der quantitativ nicht sehr dichten Korrespondenz der beiden, die ab 1904 nachweisbar ist und nach dem ersten persönlichen Treffen 1932 in Prag eine Fortsetzung bis zum Tod Thomas Manns 1955 fand, arbeitete Kilcher zwei zentrale Themen heraus. Die ersten Briefkontakte vor dem Ersten Weltkrieg kamen zustande, weil Brod sein auf Schopenhauer gründendes radikal antibürgerliches ästhetizistisches Programm in Tonio Kröger wiederzuerkennen glaubte. Mitte der 1920er-Jahre kam es nach einer langjährigen Unterbrechung zu einer Wiederaufnahme der Korrespondenz, in der fortan das »jüdisch-arabische« Projekt das zentrale Thema darstellte. Brod hatte sich zwischenzeitlich dem Zionismus zugewandt und 1925 den Roman Reubeni, Fürst der Juden veröffentlicht, während Thomas Mann den Stoff der ab 1933 erscheinenden Joseph-Romane aufgegriffen hatte. Wie der im Thomas-Mann-Archiv erhaltene Briefwechsel zeigt, sandten sich die beiden in der Folge gegenseitig ihre Werke zu und tauschten sich über einschlägige Fachliteratur aus. Dabei beachtete Thomas Mann Brods Literaturhinweise, lassen sich doch die entsprechenden Bände in der Nachlassbibliothek nachweisen. Die nicht nur ästhetische, sondern auch historische Bedeutung des Joseph-Projekts brachte Max Brod in einem Beitrag zu Thomas Manns 80. Geburtstag auf den Punkt: »Für uns in Israel hat Thomas Mann außer seiner die internationalen Gipfel stürmenden Weltbedeutung noch einen ganz besonderen Anspruch auf Liebe. Er hat uns verteidigt, er hat sich in seiner besonderen Art unser angenommen. Gerade 1933 erschien der erste Band seines Romans `Joseph und seine Brüder`, genau in dem Jahre, in dem in Deutschland die Hitler-Barbarei ausbrach. Thomas Mann hätte nun dieses ungeheure Werk [...] zur Seite legen und die Weiterführung einem geeigneteren Zeitpunkt überlassen können [...] Aber Thomas Mann legte den Joseph-Roman nicht zur Seite [...] Daß Thomas Mann das Hohelied jüdischer Schönheit und Weisheit gerade in der Geschichtsperiode angestimmt, weitergeführt und vollendet hat, in der wir Juden die verfolgteste und einflußloseste aller Nationen waren: dafür gebührt ihm unser unauslöschlicher Dank. $\ll^{5}$

Der zweite wichtige Gegenstand im Austausch zwischen Max Brod und Thomas Mann war Franz Kafka (1883-1924), seit Brod 1925 in einem Beitrag zum 50. Geburtstag Thomas Manns die Brücke zum ein Jahr zuvor verstorbenen Deutschböhmen geschlagen hatte. Thomas Mann förderte fortan die Edition der Werke Kafkas durch Brod, was ihm letzterer durch Widmungsexemplare dankte, die in der Nachlassbibliothek erhalten sind. 1938 wandte sich Brod, der in Nazi-Deutschland zunehmend in Bedrängnis geriet, mit einem Brief an Thomas Mann und bat ihn um Unterstützung bei der Emigration in die USA. Das Angebot, das Brod seinem Exilland zu machen gehabt hätte, war der Kafka-Nachlass, auf dessen Grundlage er eine Kafka-Forschungsstelle einzurichten gedachte. Thomas Mann wandte sich in der Folge tatsächlich, wenn auch letztlich ohne Erfolg, an die New York Public Library und das Hebrew 
Union College. In den erhaltenen Quellen stellen sich die Ereignisse somit anders dar, als Brod sie später in seiner Autobiografie nachzeichnete. Denn dort hatte Brod den Versuch, ihm die Flucht in die USA zu ermöglichen, als eine generöse Initiative Thomas Manns geschildert, die er aber aus zionistischen Motiven heraus nicht habe wahrnehmen wollen. Die Beziehung von Thomas Mann und Max Brod war, so das Fazit Kilchers, weder einseitig noch lässt sie sich auf allgemeine Muster des deutsch-jüdischen Verhältnisses reduzieren. Es handelte sich um eine nicht allzu dichte, aber langjährige respektvolle Bekanntschaft, die nach 1933 an persönlicher Intensität gewann. Trotz des literarischen Antisemitismus in Thomas Manns Frühwerk und späteren politischen Differenzen fanden Thomas Mann und Max Brod eine gemeinsame Basis im Humanismus und in einem europäischen Universalismus, der jüdische Literatur und Kultur nicht ausschließen, sondern integrieren wollte.

Linder, die den vielleicht germanistischsten Vortrag der Tagung lieferte, schloss eng an Kilcher an und legte dar, wie Thomas Mann die Edition der Werke Kafkas beförderte und wie intensiv er zeitlebens in und über Kafka las. Im Aufsatz Verjüngende Bücher von 1927 empfahl Thomas Mann die Kafka-Lektüre und charakterisierte dessen Schaffen erstmals als merkwürdig, seltsam, sonderbar und gerade deshalb fesselnd - ein Urteil, das er öffentlich und in seinen Tagebüchern später in nur leichter Variation regelmäßig wiederholen sollte. Die uneingeschränkte Hochachtung für den Prager Dichter, die Wertschätzung für Kafkas Texte als »fesselndes «Fremdes, die Linder insbesondere anhand von Korrespondenzstücken und Tagebuchnotizen herausarbeitete, wertete sie als schlagendes Bespiel dafür, dass Thomas Mann sich entgegen herkömmlicher Meinung auch einem Dichtertum spontan öffnen konnte, das ihm von der Grundrichtung her fremd war. Ein gemeinsam mit seinem Bruder Heinrich, Martin Buber, André Gide, Hermann Hesse und Franz Werfel unterzeichneter Aufruf des Berliner Tageblatts zur Unterstützung einer Kafka-Gesamtausgabe und das Vorwort zur amerikanischen Übersetzung von Das Schloss bilden die sichtbarsten Zeugnisse des Engagements von Thomas Mann für Kafka. Die Analyse der Tagebücher und der Lesespuren in den Kafka-Bänden, die in der Nachlassbibliothek erhalten sind, machen deutlich, dass Thomas Manns Kafka-Interpretation das Religiöse mit dem Humoristischen verschränkte, womit er auch eine Brücke zu sich selbst als Dichter schlug.

\section{Mann, Wassermann und ihr Verleger S. Fischer}

Hans Wißkirchen (Lübeck) ging dem Verhältnis zwischen Thomas Mann und Jakob Wassermann (1873-1934) nach. Der seinerzeit außerordentlich populäre Wassermann war, so Wißkirchen, ein »Genie der Freundschaft « und verstand unter Freundschaft etwas, das jenseits aller Meinungsverschiedenheiten und ästhetischen Differenzen anzusiedeln war. Thomas Mann sah in Wassermann den »deutschen Erzähler« paradigmatisch verwirklicht und anerkannte, was bei ihm selten genug vorkam, neidlos Wassermanns Überlegenheit in der »Kunst, die Leute zum Zuhören zu zwingen «. Dem Roman, wie Jakob Wassermann ihn beherrschte, sprach Thomas Mann freilich die Zukunft ab. Von einer ästhetischen Anregung oder Befruchtung als Basis der Freundschaft konnte daher keine Rede sein. Wenn das ästhetische Nebeneinander nach dem Credo Wassermanns geradezu

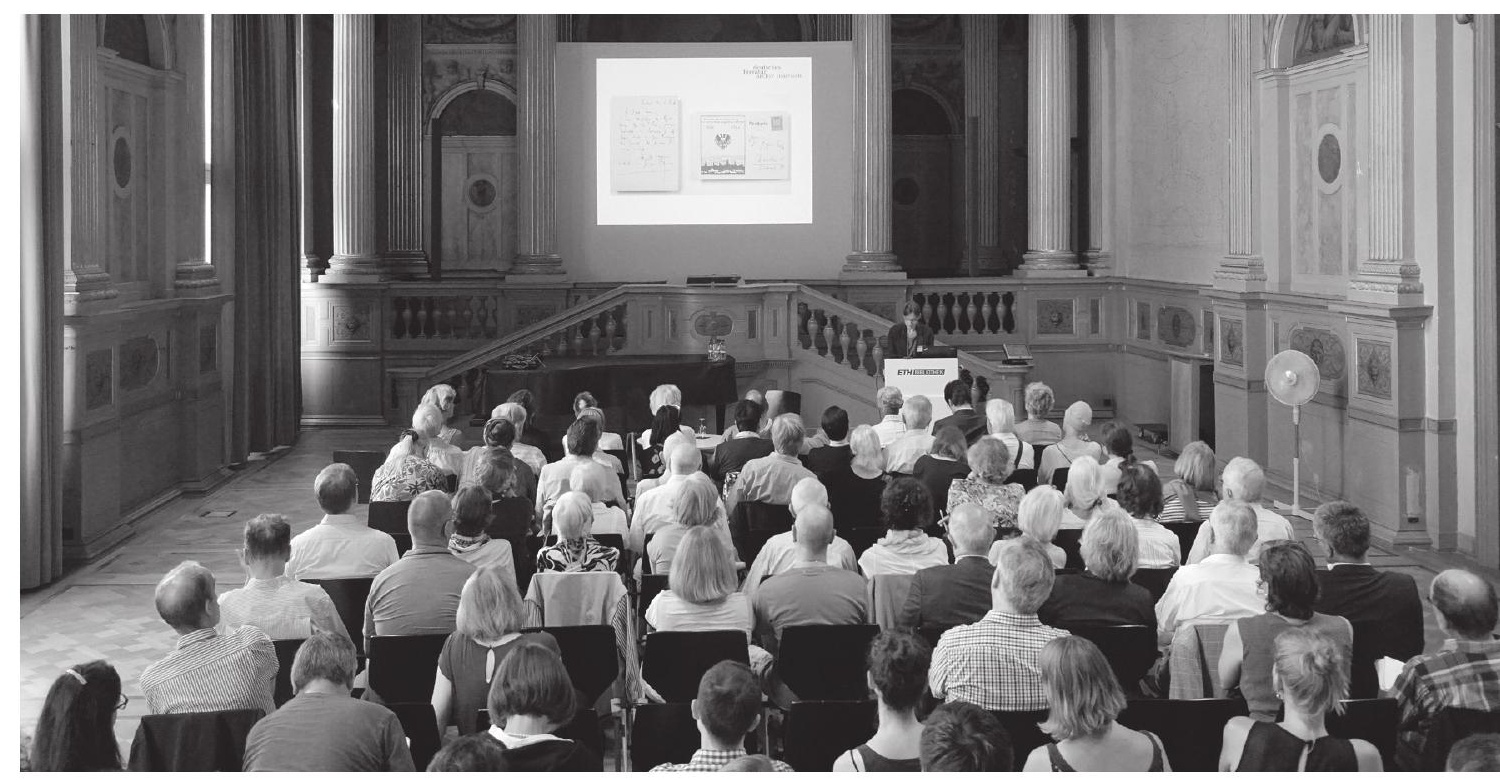

1 Die historische Semper Aula war voll besetzt.

Foto: ETH-Bibliothek, CC-BY 4.0 
eine Bedingung für das menschliche Zueinander bildete, sagt das noch nichts über die umgekehrte Perspektive, die Frage nämlich, ob die Freundschaft zwischen Wassermann und Thomas Mann letzteren in irgendeiner Hinsicht beeinflusst oder geprägt hat.

In seiner autobiografischen Schrift Mein Weg als Deutscher und Jude machte Jakob Wassermann seinen zentralen Lebenskonflikt öffentlich. Sein Versuch, sich als deutscher Schriftsteller zu etablieren, wurde insofern durch die Rezeption durchkreuzt, als dass er hauptsächlich als jüdischer Autor wahrgenommen wurde. Diese Problematik blieb Thomas Mann unverständlich, er bagatellisierte das Thema und kam zu einer beklemmenden Fehleinschätzung, etwa in einem Brief an Wassermann vom 2./3. April 1921: "Ein nationales Leben, von dem man den Juden auszusperren versuchte [...], gibt es denn das überhaupt? Deutschland zumal, kosmopolitisch wie es ist, alles aufnehmend, alles zu verarbeiten bestrebt, ein Volkstum, in dem Nordheidentum und Südsehnsucht sich ewig streiten, westliche Bürgerlichkeit und östliche Mystik sich vermischen - sollte es ein Boden sein, worin das Pflänzchen Antisemitismus je tief Wurzel fassen könnte? $^{6}$ Der Grund dafür liegt in einer identifikatorischen Lektüre. Thomas Mann konnte die Situation des Anderen nur insoweit nachvollziehen, als er darin seine eigene erkannte: Er sah und anerkannte das Außenseitertum Wassermanns, schlug aber den Bogen zu sich selbst und beschrieb Wassermann als literarischen, nicht als jüdischen Außenseiter. Noch 1933 hielt Thomas Mann an der Gleichsetzung von Literat und Jude fest und formulierte aus heutiger Sicht kaum noch nachvollziehbar: » Ein deutscher Schriftsteller - ein deutscher Märtyrer<. Man muß nicht Jude sein, um diese Erfahrung zu machen, und nur solche machen sie nicht, die gar keine Schriftsteller sind, sondern nur schreiben. « Die Engführung mit der eigenen Lebensproblematik, den eingeschränkten Blick auf Wassermann, überwand Thomas Mann bis zum Tod des Anderen 1934 nicht.

In einem zweiten zentralen Aspekt der Beziehung ist ein Einfluss Wassermanns auf Thomas Mann feststellbar. Für den Übergang von einer nationalistischen und konservativen Position zum Bekenntnis Thomas Manns zur Weimarer Demokratie trug Wassermanns Vortrag bei, den Thomas Mann am 20. November 1921 im Münchner Residenztheater gehört hatte. In der Verteidigung der »Gestalt « gegen »Gesinnung « und »Meinung « durch Wassermann erkannte Thomas Mann zu Recht eine reaktionäre Haltung und den Kampf gegen die Demokratie. Das Entscheidende ist nun, wie sich anhand von Thomas Manns Tagebuchaufzeichnungen nachvollziehen lässt, dass ihm unter anderem der Wassermann-Vortrag die Augen dafür öffnete, dass dies eine intellektuelle Sackgasse bildete, und ihn mit zur Einsicht führte, den Kampf gegen die Demokratie zu beenden und sich mit der Weimarer Republik zu versöhnen. Natürlich war es nicht allein dieser Vortrag, der die politische Einstellung Tho- mas Manns zur Demokratie veränderte, aber Wassermann hatte unbestritten seinen Anteil an dieser Entwicklung.

In einem ebenso geistreichen wie präzisen Vortrag sprach Gunilla Eschenbach (Marbach am Neckar) über das Verhältnis zwischen Thomas Mann und seinem Verleger Samuel Fischer (1859-1934). Das Archiv des S. Fischer Verlags, das sich heute im Deutschen Literaturarchiv Marbach befindet, bietet mit der erhaltenen Korrespondenz, den Fotobeständen, Buchhaltungsunterlagen und Publikationen eine günstige Ausgangslage, um diese Beziehung unter drei Aspekten darzustellen nämlich dem jüdischen, dem politisch-wirtschaftlichen sowie dem persönlichen. S. Fischer war nolens volens ein jüdischer Verlag. Der Aufstieg zu einem der renommiertesten Literaturverlage in Deutschland kann zwar als Erfolgsgeschichte jüdischer Assimilation geschrieben werden, der Verlagsgründer Samuel Fischer eignete sich jedoch aufgrund seiner Physiognomie und seines Akzents als Zielscheibe für diejenigen, die Stereotypen des Jüdischen und antisemitische Vorurteile pflegten. Beim Tod Fischers 1934 waren die Nachrufe in den regimenahen Zeitungen tendenziös und antisemitisch geprägt. So weit ging die Gedenkschrift des Verlags selbstverständlich nicht, aber auch hier waren Nase und Oberlippe auf einer Fotografie des Verstorbenen so retuschiert, dass die »jüdische Physis « aufgehoben wurde, und in den Texten fanden sich sprachliche Anbiederungen an den Nationalsozialismus. Thomas Mann war der einzige unter den Kondolierenden, der das Judentum seines Verlegers im Gedenkbuch nicht nur andeutete, sondern offen und zugleich positiv erwähnte: Die Schriftkultur des Judentums habe in Fischer diejenige Liebe zum Buch wachsen lassen, die ihn zu einem großen Verleger gemacht habe.

Anhand der Unterlagen im S. Fischer-Verlagsarchiv lässt sich zeigen, wie sich die Machtergreifung der Nationalsozialisten 1933 auf die Beziehung zwischen Verlag und Autor auswirkte. Zwei Beobachtungen sind besonders wichtig. Zum einen versuchte der Verlag aus ökonomischen Gründen, Thomas Mann für den deutschen Markt zu retten, und bat ihn deshalb mehrfach, den Verkauf seiner Bücher im Deutschen Reich nicht durch unbedachte politische Äußerungen zu gefährden. Zum anderen zeigen die überlieferten Honorar- und Lizenzabrechnungen einen Bruch in den finanziellen Transaktionen. Auch wenn vor weiteren Untersuchungen unklar bleibt, ob die Abrechnungen zugunsten Thomas Manns frisiert, Gelder auf alternativen Wegen transferiert bzw. auf Auslandskonten einbezahlt, oder aber von den deutschen Behörden beschlagnahmt wurden, steht zweifelsfrei fest, dass sich die politische Repression unmittelbar auf dem Bankkonto Thomas Manns niederschlug. Die persönlichen Beziehungen zu Thomas Mann pflegte Samuel Fischer weiter, als er die Verlagsgeschäfte aus gesundheitlichen Gründen bereits weitgehend an seine 


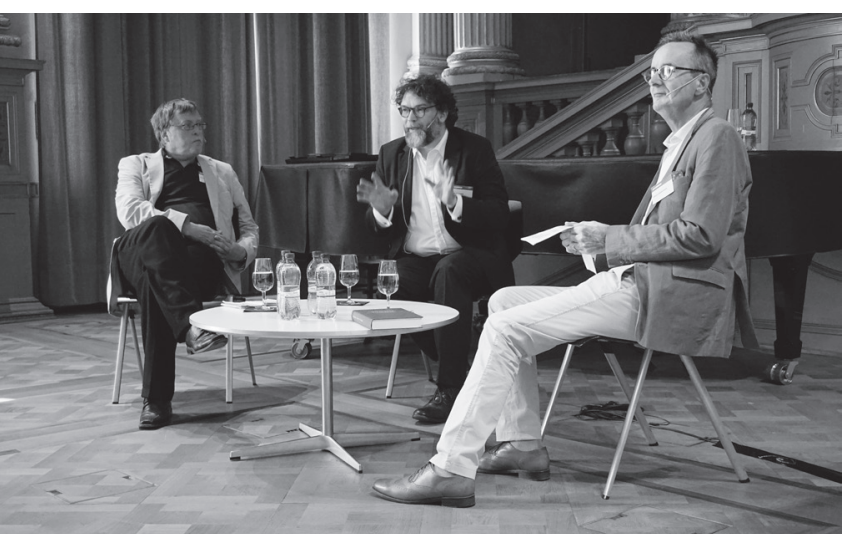

2 Franz Zeder, Oliver Matuschek und Vittorio Klostermann auf dem Podium (v. li.) Foto: ETH-Bibliothek, CC-BY 4.0

Erben weitergegeben hatte. Diese Beziehungen scheinen weder besonders herzlich noch auffallend geschäftsbezogen gewesen zu sein. Dies fügt sich in die Strategie Fischers, Beziehungen zu Autoren langfristig zu gestalten, indem er diese in Gesamtausgaben bündelte, an seine eigenen Zeitschriften band und Jubiläen systematisch für Sonderausgaben nutzte, um beim Lesepublikum neue Aufmerksamkeit für einen Autor zu wecken.

\section{Mann und Zweig}

In seinem Jubiläumsjahr legte das Thomas-MannArchiv die wissenschaftliche kommentierte Edition des Briefwechsels zwischen Thomas Mann und Stefan Zweig (1881-1942) vor. ${ }^{8}$

Dieser Band bildete den Ausgangspunkt des angeregten Podiumsgesprächs zwischen Mitherausgeber Franz Zeder (Graz) und dem Zweig-Biografen Oliver Matuschek (Hannover), das Verleger Vittorio Klostermann (Frankfurt am Main) moderierte. Die Gesprächspartner arbeiteten heraus, dass sich nicht nur Thomas Mann im Laufe seines Lebens vom nationalistischen Propagandisten zum Anhänger der Demokratie entwickelte, sondern dass auch Stefan Zweigs politische Haltung einem Wandel unterlag. Erst das Fronterlebnis 1915 nach einer freiwilligen Meldung zur Armee machte ihn zu dem kompromisslosen Pazifisten, der er später war. Nimmt man den Briefwechsel als Ganzes, war das jüdische Thema nicht prägend. Gleichwohl lassen sich weitere Aufschlüsse über Stefan Zweigs Selbstverständnis als »deutschen Juden« und Internationalisten gewinnen. Unabhängig davon beleuchtet die nun edierte Korrespondenz die Hintergründe mancher Episode neu, etwa des Bruchs zwischen Richard Strauss und seinem Libretto-Autor Stefan Zweig, den Zweigs mehrwöchiger Zürcher Aufenthalt anlässlich von Thomas Manns 60. Geburtstag mitverursacht hatte.

Insgesamt führte die Tagung am Beispiel des ThomasMann-Archivs die eminente Rolle von Literaturarchiven generell vor Augen. Die dort überlieferten Textvarianten, Korrespondenzen und Selbstzeugnisse, die in Form von persönlicher Bibliothek und Lesespuren materialisierten Einflüsse auf einen Autor sowie die Dokumentation seiner Rezeption weisen weit über die Literaturwissenschaft im engeren Sinne hinaus. Sie bilden eine unabdingbare Grundlage, die Geistes-, Sozial- und Politikgeschichte ihrer jeweiligen Epoche zu schreiben. Indem sie das 1956 gegründete Thomas-Mann-Archiv in den letzten Jahren als eines der informationswissenschaftlich modernsten Literaturarchive positioniert hat, nimmt die ETH-Bibliothek ihre Verantwortung zugunsten der Forschung wahr.

Eine Publikation der Tagungsbeiträge im Thomas Mann Jahrbuch 2018 ist geplant.

\section{Anmerkungen}

1 SPRECHER, Thomas, Hrsg., 2006. Im Geiste der Genauigkeit. Das Thomas-Mann-Archiv der ETH Zürich 1956-2006. Frankfurt/Main: Klostermann. BEDENIG, Katrin, 2015. Nachlass und Archive. In: Andreas BLÖDORN und Friedhelm MARX, Hrsg. Thomas Mann Handbuch. Leben, Werk, Wirkung. Stuttgart: Metzler, S. 373-376.

2 THOMAS-MANN-ARCHIV, 2016. Thomas-Mann-Archiv Online [online]. Zürich: ETH Zürich [Zugriff am 08.10.2016]. Verfügbar unter: www.online.tma.ethz.ch

3 GASSER, Michael, 2016. Innovating access to ETH-Library's Thomas Mann Archive: A project report. In: IFLA Journal [online]. 42(2), S. 134-139 [Zugriff am 08.10.2016]. Verfügbar unter: DOI: 10.1177/0340035216647392

4 P3 SNF FORSCHUNGSDATENBANK, 2016. Produktive Lektüre [online]. Thomas Manns Nachlassbibliothek. Bern: Schweizerischer Nationalfonds [Zugriff am 08.10.2016] Verfügbar unter: http://p3.snf.ch/project-162384

5 MANN, Thomas, 1974. [Zur jüdischen Frage]. In: Thomas MANN, Nachträge. Frankfurt/Main: Fischer, S. 470. Gesammelte Werke in dreizehn Bänden. 13.

6 MANN, Thomas, 1988. Briefwechsel mit Autoren. Hrsg. v. Hans WYSLING. Frankfurt/Main: Fischer, S. 594 f.

7 MANN, Thomas, 1974. Tischrede auf Wassermann. In: Thomas MANN, Reden und Aufsätze 2. 2., durchges. Aufl. Frankfurt/Main: Fischer, S. 452. Gesammelte Werke in dreizehn Bänden. 10.

8 MANN, Thomas, 1988. Briefwechsel mit Autoren. Hrsg. v. Hans WYSLING. Frankfurt/Main: Fischer, S. 477 f.

9 MANN, Thomas, 1974. Zum Geleit (Für Martha Karlweis Jakob Wassermann<). In: Thomas MANN, Nachträge. Frankfurt/Main: Fischer, S. 836. Gesammelte Werke in dreizehn Bänden. 13.

10 BEDENIG, Katrin und Franz ZEDER, Hrsg., 2016. Thomas Mann - Stefan Zweig. Briefwechsel, Dokumente und Schnittpunkte. Frankfurt/Main: Klostermann.

\section{Der Verfasser}

Dr. Stefan Wiederkehr, ETH Zürich, ETH-Bibliothek, Leitung Sammlungen und Archive, Rämistraße 101, CH-8092 Zürich, Telefon +4144632 2183, stefan.wiederkehr@library.ethz.ch 